\title{
ALGUNAS CARENCIAS DETECTADAS EN LOS ESTUDIANTES QUE INGRESAN A INTEC Y RECIBEN FÍSICA GENERAL EN SUS CARRERAS. CONSIDERACIONES SOBRE EL DESARROLLO Y USO DEL VIDEO JUEGO EN LA ENSEÑANZA DE LA FÍSICA
}

Some shortcomings with students who enter and receive INTEC General Physics in their careers. Considerations on the development and use of video games in the teaching of physics

\section{José Antonio AceitunoI Marcos Marmolejos ${ }^{\mathrm{II}}$ Evangelista Marte ${ }^{I I I}$ Pablo Bolívariv Alejandro AceitunoV}

\footnotetext{
I Profesor-investigador del INTEC. Email: jose.aceituno@intec.edu.do

II Es egresado de INTEC de la carrera de Ingeniería Electrónica y Telecomunicaciones. Email: marco.marmolejos@gmail.com

III Es estudiante de término de la carrera de Ingeniería Civil del Instituto Tecnológico de Santo Domingo (INTEC). Email: evangelista.399@gmal.com

IV Estudiante de término de la carrera de Ingeniería en Sistemas del Instituto Tecnológico de Santo Domingo. Email: pablobolivarjoa@gmail.com

v Es egresado de INTEC. Licenciado en Matemáticas con concentración en Estadísticas y Ciencias Actuariales. Email: aceitunoalvarez@hotmail.com
} 
Resumen: En este artículo se plasman los resultados de una investigación que se realiza para detectar las principales necesidades educativas que presentan los alumnos que ingresan a INTEC y que reciben la asignatura Física General en su carrera. Se aplican las técnicas comprendidas en el Modelo de Análisis de Necesidades Socio Educativas (ANISE), aprovechando su versatilidad y potencialidades que se ajustan a las pretensiones de este proyecto. Además se propone como una posible solución a las carencias detectadas, pues se pasa a la elaboración y utilización de un ambiente interactivo en tres dimensiones (videojuego), como elemento didáctico que permite incidir sobre las dificultades que los propios alumnos explicitan, como resultado de las técnicas aplicadas en este trabajo.

Palabras clave: Necesidades educativas, estudiantes, Física general, uso del video juego, enseñanza de la Física, ambiente interactivo, INTEC.

Abstract: In this article the results of research conducted to identify key educational needs that the students who enter INTEC and receiving General Physics course are reflected in his career. Apply the techniques included in the analysis model Socio Educational Needs (ANISE), taking advantage of its versatility and potential to match the scope of this project. In addition it is proposed as a possible solution to the shortfalls, as it is passed to the development and use of an interactive environment in three dimensions (video game), as a didactic element enabling impact on the difficulties the students themselves explicit, as a result of techniques applied in this work.

Keywords: Educational needs, students, general physics, use of video game physics teaching, interactive environment, INTEC. 
Algunas carencias detectadas en los estudiantes que ingresan a INTEC y reciben Física General en sus carreras

\section{Introducción}

Las universidades se nutren de estudiantes que provienen del bachillerato y de la enseñanza tecnológica, con una formación acorde a los programas de estudio para ese nivel. Tradicionalmente, como es conocido, se han confrontado dificultades con los alumnos del primer año de las carreras universitarias cuando se enfrentan a los contenidos de la Física, pues se supone que la estructura cognitiva que ellos presentan resulta adecuada y garantice el soporte de los nuevos conocimientos y habilidades que deben apropiarse. Esta base debe garantizar el paso adecuado a un nivel cualitativamente superior de su formación, pero en realidad no sucede así.

\subsection{ANTECEDENTES}

Según el Informe de la Comisión Internacional para el Desarrollo Estratégico de la República Dominicana (noviembre de 2010), bajo la dirección de Jacques Attali, los resultados globales del sistema escolar clasifican a la República Dominicana entre los Estados menos eficaces de la región. Las principales carencias reveladas en los estudios comparativos internacionales muestran que los alumnos dominicanos son particularmente malos en conocimiento de la lengua española y de las matemáticas. (Attali, et al., 2010).

Por otro lado, en el Reporte Harvard, denominado Construyendo un mejor futuro para la República Dominicana: Herramientas para el desarrollo, se explicita que el percentil 90 de los estudiantes dominicanos (el $10 \%$ con los mejores estudiantes del país) serían estudiantes del percentil 10 (10\% de los estudiantes con menor rendimiento) en los países que encabezan el ranking de resultados académicos en países con mejores sistemas educativos. (Hausmann, et al., 2012). En otras palabras, en promedio, los "estudiantes estrellas" de República Dominicana serían estudiantes con problemas de aprendizaje en países como Corea del Sur, Holanda y Canadá. 
José Antonio Aceituno, Marcos Marmolejos, Evangelista Marte, Pablo Bolivar, Alejandro Aceituno

La gravedad del problema es tal que el porcentaje de estudiantes dominicanos que estarían por encima del Nivel 1 en la prueba PISA, propuesto en Filmer, Hassan y Pritchett (2006) como el Objetivo de Aprendizaje del Milenio Mínimo (OAMmin), es de apenas $26 \%$ en matemáticas, $43 \%$ en lectura y $50 \%$ en ciencias.

En el caso específico de la Física Básica, exactamente en los contenidos referidos a la Cinemática y a la Dinámica, en el titulado Informe Física General I (Arias, M.; Romero, V. \& Pérez, V., 2011), que involucra una población representativa de la Universidad Autónoma de Santo Domingo (UASD) y de Instituto Tecnológico de Santo Domingo (INTEC), se presentan los resultados que citamos a continuación:

- INTEC: porcentaje de respuestas correctas de cinemática, utilizando un examen internacional (Beichner, R. J. (1994). Testing student interpretation of kinematics graph. Am. J. Phys., 62(8), 751-753).

Entrada $=20.7 \%$, Salida $=32.7 \%$

- INTEC: porcentaje de respuestas correctas de dinámica, utilizando un examen internacional (Hestenes, D.; Wells, M. and Swackhamer, G. Phys. Teach. 30, 141, 1992).

Entrada $=24 \%$, Salida $=27 \%$

- Universidad Autónoma de Santo Domingo (UASD): porcentaje de respuestas correctas de cinemática, utilizando un examen internacional (Beichner, R. J. "Testing student interpretation of kinematics graph" 1994. Am. J. Phys.Vol. 62, N. ${ }^{\circ}$ ).

Entrada $=17 \%$, Salida $=18.2 \%$

- Universidad Autónoma de Santo Domingo (UASD): porcentaje de respuestas correctas de dinámica, utilizando un examen internacional (Hestenes, D.; Wells, M. and Swackhamer, G. Phys. Teach. 30, 141 1992).

Entrada $=18 \%$, Salida $=20.3 \%$. 
Como puede observarse, los resultados en ambas instituciones están muy por debajo de lo que aspiramos (al menos $70-80 \%$ en la salida), y es importante que busquemos alternativas a los métodos tradicionalmente utilizados para resolver esta problemática.

En ese sentido, nos enfocamos en investigar sobre las principales carencias que presentan los estudiantes de las diferentes carreras del INTEC, que reciben la Física Básica como parte de su formación, con la intensión de proponer soluciones a partir del desarrollo de ambientes interactivos en $3 \mathrm{D}$, que permitan mejorar la calidad de la enseñanza de la Física a los estudiantes que concluyen el bachillerato e ingresan a la Universidad.

La integración del uso de las nuevas tecnologías a la enseñanza es una exigencia de la actualidad, y pretendemos hacerlo de una manera muy peculiar, poniendo en manos de aprendices y enseñantes, herramientas que permitirán hacer del proceso de enseñanza-aprendizaje una actividad placentera, donde el estudiante aprende mientras juega y va venciendo los retos que se le presentan construyendo las soluciones a partir del análisis de las situaciones problémicas.

\section{Marco teórico}

El concepto de estructura cognitiva que se asume en este proyecto se limitará a la forma en que cada individuo tiene organizados sus conocimientos y habilidades, así como la relación que existe entre ellos (Lejter de Bascones, 1990: 26).

Al intentar modificar la estructura cognitiva de los estudiantes, se le están propiciando nuevos conocimientos y desarrollándoles nuevas habilidades sobre la base de los conocimientos y las habilidades que ya posee, garantizando que se logre una relación armónica entre lo "nuevo" y lo "primitivo". 
José Antonio Aceituno, Marcos Marmolejos, Evangelista Marte, Pablo Bolivar, Alejandro Aceituno

Es de vital importancia eliminar la discontinuidad que se produce entre ambos niveles de enseñanza, ya sea porque los alumnos carezcan de los conceptos previos necesarios, o si los posee, tales conceptos pueden ser errados; en este caso, la nueva información no se retiene y el estudiante se limita a memorizar como cuando "aprende" fórmulas de la propia Física, que no es más que el llamado aprendizaje mecánico. Todo esto dificulta, de manera considerable, el proceso de apropiación de los nuevos conocimientos y la adquisición de nuevas habilidades.

Para resolver esta situación se han utilizado los "cursos de nivelación", en los que se le suministra a los estudiantes la información y los conocimientos que necesitan posibilitando un desarrollo adecuado del proceso de aprendizaje en el que están involucrados. Sin esto es muy difícil obtener buen rendimiento académico de los futuros profesionales.

Los "cursos de nivelación" no son la única vía para lograr este objetivo, existen varias formas, y la utilización ambientes virtuales de aprendizaje se revela como un método novedoso y eficaz, pues sus potencialidades comunicativas son ilimitadas, así como la formación más integral que proporciona a los estudiantes la utilización de la máquina computadora en el proceso de aprendizaje, máxime en estos tiempos donde la automatización y la cibernética se van haciendo indispensables en todas las esferas de la vida.

Este investigación se apoya esencialmente en la teoría del aprendizaje significativo (Ausubel, 1990; González y Novak, 1993; Noger C, 1997; Novak y Gowin, 1992) como marco teórico para el desarrollo de una tecnología educativa destinada a facilitar una interacción más efectiva entre la instrucción y el aprendizaje, pues de esta forma se garantiza que se aprenda de forma interactiva, deja un margen muy pequeño para el aprendizaje mecánico y contribuye al desarrollo de determinadas habilidades por parte de los alumnos, donde se destaca la de generalizar, específicamente en el momento en que comienzan a recibir las asignaturas de Física, que forman parte del plan de estudios de las carreras universitarias. 
El uso de organizadores previos (Ausubel, ctdo. por Letjer de Bascones 1990), es una de las técnicas instruccionales utilizadas para favorecer la transición cognitiva del concepto intuitivo al concepto científico, la adquisición de conceptos nuevos y la formación de estructuras conceptuales apropiadas para la solución de problemas.

El principal aporte de la teoría de Ausubel en el constructivismo, es su modelo de enseñanza por exposición, para promover el aprendizaje significativo en lugar del aprendizaje de memoria. Este modelo consiste en explicar o exponer hechos o ideas. Este enfoque es de los más apropiados para enseñar relaciones entre varios conceptos, pero antes los alumnos deben tener algún conocimiento de dichos conceptos.

Otro aporte al constructivismo son los organizadores previos, los cuales sirven de apoyo al alumno frente a la nueva información, funciona como un puente entre el nuevo material y el conocimiento actual del alumno. Estos organizadores pueden tener tres propósitos (Ausubel, 1978): dirigir su atención a lo que es importante del material; resaltar las relaciones entre las ideas que serán presentadas y recordarle la información relevante que ya posee.

Los organizadores anticipados se dividen en dos categorías:

- Comparativos: activan los esquemas ya existentes, es decir, le recuerdan lo que ya sabe pero no se da cuenta de su importancia. También puede señalar diferencias y semejanzas de los conceptos.

- Explicativos: proporcionan conocimiento nuevo que los estudiantes necesitarán para entender la información que subsiguiente. También ayudan al alumno a aprender, especialmente cuando el tema es muy complejo, desconocido o difícil; pero estos deben ser entendidos por los estudiantes para que sea efectivo. 
José Antonio Aceituno, Marcos Marmolejos, Evangelista Marte, Pablo Bolivar, Alejandro Aceituno

Los estudiantes que tienen dificultades para poder ordenar los problemas complicados, se ven limitados no tanto por su falta de agilidad matemática y dominio de las Leyes Físicas, sino por su incapacidad de poder construir modelos que permitan visualizar, comprender mejor y generalizar los nuevos conocimientos y habilidades adquiridos.

\section{Metodología}

Consideramos que por los intereses y las características de esta investigación, el Modelo de Análisis de Necesidades de Intervención Socioeducativa (ANISE).

Paz Pérez-Campanero, (1991), es el que mejor se ajusta al propósito de la investigación, pues se desarrolla mediante la recopilación de datos sobre una serie de problemas propios de una población, para después tomar decisiones sobre la ejecución o no, de un programa de intervención, determinar su envergadura, proponer los objetivos y fundamentar el proceso de planificación e implementación, coincidente con el objetivo general que se declara para esta investigación.

Otra razón importante que determinó la filiación a este modelo para la evaluación de necesidades educativas, es que se presenta como un compendio de herramientas, que se pueden considerar como un sistema bien organizado de para el trabajo de campo.

De acuerdo con el alcance que marca el objetivo de esta investigación, corresponde realizar solo las dos primeras etapas que describe el modelo de referencia: Reconocimiento y Diagnóstico. Por su parte, la Toma de Decisiones requiere la prolongación del estudio iniciado, con posibilidades de realizar una intervención educativa, que deriva de esta fase.

En la etapa de reconocimiento se tratan de determinar las situaciones desencadenantes de la situación que se estudia, se seleccionan 
las herramientas o instrumentos para la obtención de datos, además de la búsqueda de fuentes de información que puede hacerse a partir de las personas implicadas, además de otras fuentes.

Por su parte en la etapa de diagnóstico se identifica la situación actual y se establece la situación deseable, se analiza e identifican las causas que provocan las discrepancias entre la situación actual y la deseable en términos de condiciones existentes y requeridas. También se identifican los sentimientos que producen en los implicados esas discrepancias. Además en esta etapa se puede definir el problema en términos claros y precisos.

Ya en la etapa de toma de decisiones, aunque no se desarrolla en esta investigación, se deben priorizar los problemas definidos y proponer las soluciones, evaluando su costo, impacto y viabilidad.

Para esta investigación en concreto las etapas se desarrollaran de la manera siguiente:

ETAPA DE RECONOCIMIENTO

Objetivo:

- Realizar el reconocimiento de la situación que se tiene en cuanto los conocimientos de Física que tienen los estudiantes que ingresan nuevos a la Universidad INTEC.

Técnicas y procedimientos:

Se utiliza la herramienta Grupo Central.

ETAPA DE DiAGNÓSTICO:

Objetivo:

- Determinar cuáles son las causas que desencadenan las discrepancias entre la situación actual y la situación deseada y evaluar cuáles son los sentimientos que provocan en los implicados, estas discrepancias. 
José Antonio Aceituno, Marcos Marmolejos, Evangelista Marte, Pablo Bolivar, Alejandro Aceituno

Técnicas y procedimientos:

Se utilizan las herramientas Grupo Central y Sondeo de Problemas.

Con los datos que proporcionen estas herramientas, además de la bibliografía revisada, se realiza un análisis que lleve a diseñar y construir la primera versión de un video juego que permita ir dando solución a las carencias detectadas.

\section{Análisis y discusión de los resultados}

\subsection{Etapa de reconocimiento}

\subsubsection{REUNIÓN PREPARATORIA}

La reunión tiene como propósito, buscar una aproximación a las dificultades que se han presentado en el aprendizaje de las leyes fundamentales de la Física, desde la perspectiva de los alumnos y seleccionar a los estudiantes que formarán el "Grupo Central" una de las técnicas que se utilizarán para desarrollar esta investigación.

Fueron convocados estudiantes de una sección de Física I, en un horario donde no tenían ninguna actividad. De un total de 40 asistieron de forma voluntaria 31, al aula 301 del Edificio García de la Concha (GC-301), del Instituto Tecnológico de Santo Domingo (INTEC).

Este grupo es escogido, precisamente porque comienzan a recibir por primera vez en su carrera, la asignatura de Física I, que contempla parte de los fundamentos de la Mecánica Newtoniana.

La reunión comienza con una invitación del profesor investigador a hablar sobre "El aprendizaje de la Física en la enseñanza media". De forma inmediata comienzan a surgir criterios desordenadamente sobre diferentes aspectos. En un tiempo breve, ya de manera organizada, se fueron aclarando los diferentes tópicos. 
La gran cantidad de criterios emitidos se fueron agrupando en la medida que tuvieran relación entre sí o trataran sobre el mismo aspecto. Las intervenciones se fueron sintetizando en la pizarra, quedando los criterios siguientes:

- Predominio de la solución de Problemas sobre la discusión teórica de las Leyes Físicas.

- Aprendizaje memorístico a partir de la utilización de fórmulas que se usan mecánicamente.

- Poca vinculación con la vida cotidiana.

- Escasas Prácticas de Laboratorio.

- Insuficientes horas de clases en los diferentes niveles.

Posteriormente se pasó a seleccionar a los estudiantes que formarían parte del "Grupo Central". Para ello, el profesor investigador les pidió que propusieran a 8 ó 10 de los compañeros que consideraran capaces de representar a todo el colectivo en reflexiones más profundas acerca de los criterios "definitivos" que habían quedado plasmados en la pizarra, como reflejo de las principales dificultades que están incidiendo negativamente en el aprendizaje de la Física.

De manera espontánea comenzaron las propuestas en el orden siguiente:

M.F.E., M.M.L., K.G.C., M.M.O., S.H.R., M.M.V., O.L.E., G.M.H. y N.G.S.

Los argumentos que sustentan las proposiciones se relacionan con la capacidad de análisis, el carácter, sensibilidad y compromiso con el grupo y buen rendimiento académico.

Todos aceptaron la responsabilidad y el grupo entendió que con ellos era suficiente para llevar a cabo un buen análisis de la situación planteada y que esos 9 compañeros eran capaces de representar al grupo en su totalidad. 
José Antonio Aceituno, Marcos Marmolejos, Evangelista Marte, Pablo Bolivar, Alejandro Aceituno

Se da por terminada la reunión y se le pide a los 9 seleccionados que se queden para fijar la fecha y la hora para realizar el "Grupo Central". Se acordó que lo más conveniente era reunirse la próxima semana, donde todos estaban con tiempo libre y además tenían la posibilidad de ir pensando acerca de los tópicos que servirían de eje fundamental para las reflexiones e intercambios.

\subsubsection{GRUPO CENTRAL}

El "Grupo Central" se desarrolló después de transcurridos 7 días desde que se celebró la reunión preparatoria, como se había acordado, en el horario libre que tenían y en la propia aula 301 del edificio GC del INTEC.

Asistieron los 9 seleccionados y se comenzó a la 1:00 pm (13:00 horas). El local fue acondicionado al efecto, colocando las sillas y mesas para cada participante, alrededor de un círculo imaginario, lo que propiciaba que todos pudieran observarse de frente y en todo momento. Cada puesto de trabajo posee las condiciones mínimas como para que cada sujeto pueda tomar notas y hacer apuntes cómodamente si así lo desea. Las condiciones de Iluminación, ventilación y la privacidad son adecuadas.

El profesor investigador registrará el desenvolvimiento de la reunión usando una cámara de vídeo, además de los apuntes que tomará durante el desarrollo de la actividad.

La cámara de vídeo fue colocada sobre un trípode, justamente detrás de la posición que ocupa el profesor en el "circulo", a una distancia y a una altura prudenciales, que permitiese que, en un plano general se observara y se escuchara al grupo en su totalidad, pero que a su vez no constituyera un elemento disociador ni entorpecedor dentro del contexto, como se muestra en el esquema siguiente. 


\section{Esquema N. ${ }^{\circ} 1$}

Ubicación en el aula de los participantes en el Grupo Central

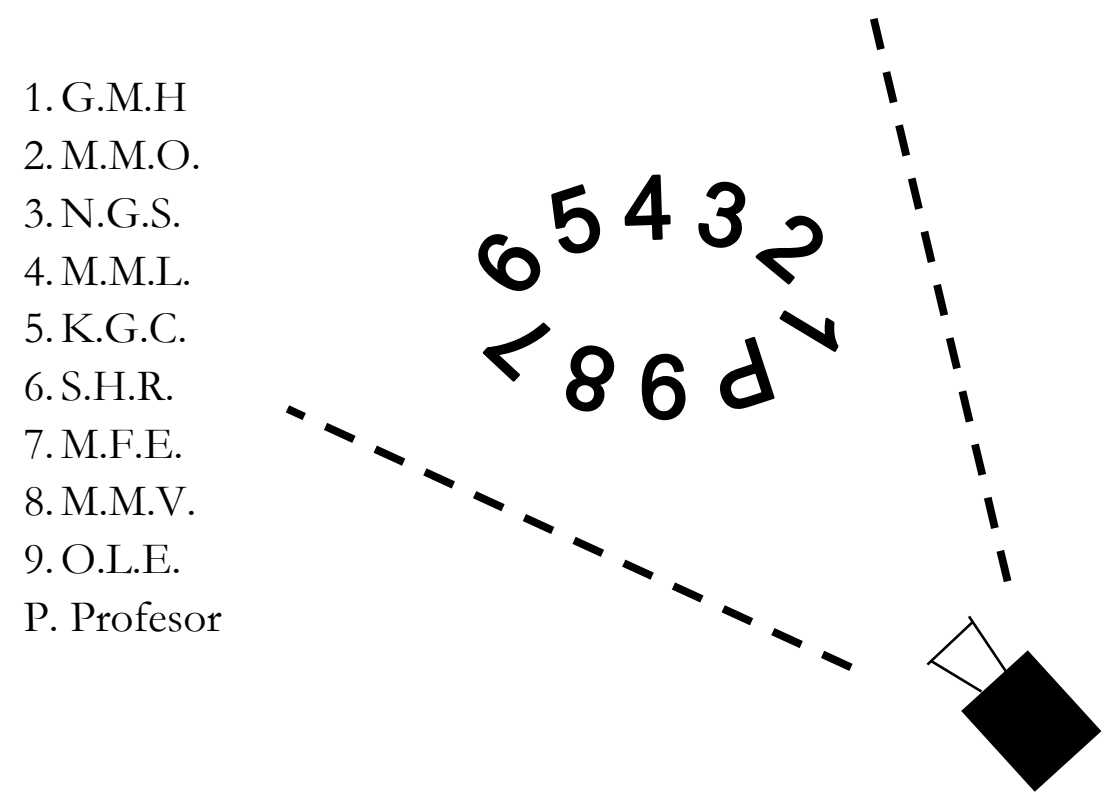

El profesor en calidad de facilitador de la discusión, menciona en forma de recordatorio, los aspectos que se analizaron en "la reunión preparatoria”, a la que se convocó el grupo.

No se manifestó ningún comportamiento que denotara resistencia a participar, de forma inmediata y espontánea se sucedieron varias intervenciones, sobre varios aspectos, que fueron tratados en diferentes momentos de la reunión. Algunos de los aspectos fueron retomados en más de una ocasión, en la medida que los participantes consideraron oportuno relacionarlo con lo que se discutía en ese momento.

Con el objetivo de facilitar la comprensión por parte del lector de la información que se plasma en estas páginas, se reflejan a continuación los tópicos que fueron tratados y en el orden en que fueron apareciendo, resumidos en lo que pudiéramos llamar, "El Guión de la Discusión”. 
José Antonio Aceituno, Marcos Marmolejos, Evangelista Marte,

Pablo Bolivar, Alejandro Aceituno

\subsubsection{GUIÓN DE LA DISCUSIÓN}

1) Insuficiente discusión teórica sobre los fenómenos físicos y sus leyes.

2) Poca o ninguna utilización de experimentos demostrativos en el aula.

3) Solución de problemas a partir de la utilización de fórmulas predeterminadas para calcular cada variable especifica

4) No se relacionan suficientemente los contenidos que se imparten con los fenómenos de la vida cotidiana.

5) Se realizan muy pocas Prácticas de Laboratorio.

6) Existen muy pocas horas dedicadas a la impartición de la Física.

Después de haber visualizado el vídeo de la reunión en su totalidad, nos pareció oportuno hacer una tabla que agrupara los tópicos y recogiera el número de intervenciones realizadas en cada uno de ellos, por parte de los sujetos (estudiantes) que participan. Se presenta además, la cantidad de intervenciones hecha por el profesor en cada uno de los tópicos.

Tabla N. ${ }^{\circ} 1$

Cantidad de intervenciones hechas por tópicos

\begin{tabular}{|l|c|c|}
\hline \multicolumn{1}{|c|}{ Tópicos } & $\begin{array}{c}\text { Cantidad de } \\
\text { Intervenciones } \\
\text { de los alumnos }\end{array}$ & $\begin{array}{c}\text { Cantidad de } \\
\text { intervenciones } \\
\text { del profesor }\end{array}$ \\
\hline $\begin{array}{l}\text { Insuficiente discusión teórica sobre } \\
\text { los fenómenos físicos y sus leyes }\end{array}$ & 9 & - \\
\hline $\begin{array}{l}\text { Poca o ninguna utilización de expe- } \\
\text { rimentos demostrativos en el aula }\end{array}$ & 14 & 3 \\
\hline
\end{tabular}




\begin{tabular}{|l|c|c|}
\hline \multicolumn{1}{|c|}{ Tópicos } & $\begin{array}{c}\text { Cantidad de } \\
\text { Intervenciones } \\
\text { de los alumnos }\end{array}$ & $\begin{array}{c}\text { Cantidad de } \\
\text { intervenciones } \\
\text { del profesor }\end{array}$ \\
\hline $\begin{array}{l}\text { Solución de problemas a partir } \\
\text { de la utilización de fórmulas pre- } \\
\text { determinadas para calcular cada } \\
\text { variable especifica }\end{array}$ & 12 & 1 \\
\hline $\begin{array}{l}\text { No se relacionan suficientemente } \\
\text { los contenidos que se imparten } \\
\text { con los fenómenos de la vida co- } \\
\text { tidiana }\end{array}$ & 31 & 4 \\
\hline $\begin{array}{l}\text { Se realizan muy pocas Prácticas } \\
\text { de Laboratorio }\end{array}$ & 16 & \\
\hline $\begin{array}{l}\text { Existen muy pocas horas dedica- } \\
\text { das a la impartición de la Física }\end{array}$ & 7 & \\
\hline
\end{tabular}

A manera de resumen, podemos referirnos a cada uno de los tópicos que se presentan en la tabla anterior (Tabla N. $\left.{ }^{\circ} 1\right)$.

Por ejemplo, en el primer tópico, los planteamientos estuvieron dirigidos a que en las clases de física que recibieron se mencionaban las leyes, se planteaban las ecuaciones y no se hacía una demostración de cómo llegar a esa ley, lo que se relaciona muy estrechamente con lo que se planteó en el tópico 3, donde explicitan la necesidad de que no se resuelvan problemas a partir de fórmulas predeterminadas, sino que sean capaces de obtener esas fórmulas en el proceso de discusión teórica del fenómeno en sí, y que a partir de ahí, se apliquen a las situaciones problémicas específicas que deben resolver. 
José Antonio Aceituno, Marcos Marmolejos, Evangelista Marte, Pablo Bolivar, Alejandro Aceituno

Los tópicos 2, 4 y 5 aglutinan el mayor número de intervenciones, relacionadas todas con la vinculación de los contenidos teóricos que se imparten, con la práctica diaria. La necesidad de la explicación de las diferentes leyes de la Física, a partir de experimentos demostrativos en el aula, y también la posibilidad de comprobar esas Leyes a través de la realización de Prácticas de Laboratorio, donde cada participante, manipule diferentes equipos, haga mediciones y realice cálculos, que les permitan comprobar la veracidad de las leyes estudiadas, son elementos que los participantes defienden con mucha fuerza.

Es importante destacar la tendencia que se manifestó a favor de la vinculación que debe existir entre lo que se imparte en el aula y los fenómenos de la vida cotidiana.

En nuestra opinión, resulta interesante ver cómo se comportan los porcientos de las intervenciones por cada tema y cómo se distribuyeron a lo largo del tiempo que duró la reunión. Con ese fin se elaboró un gráfico donde se plotean los valores de la cantidad de intervenciones en las ordenadas y el tiempo transcurrido en las abscisas.

Los datos para la elaboración de dicho gráfico se organizaron en la tabla N. ${ }^{\circ}$ 2. Es conveniente aclarar que los valores del tiempo transcurrido en cada tema tratado, son aproximados, teniendo en consideración, que no es significativo para nuestros propósitos, el análisis del tiempo exacto en que se sucedieron los comentarios y reflexiones, y sí ubicarnos temporalmente en el momento y la cantidad aproximada de tiempo que se utilizó, para establecer alguna comparación que puede resultar útil en un análisis posterior. 
Algunas carencias detectadas en los estudiantes que ingresan a INTEC y reciben Física General en sus carreras

Tabla N. ${ }^{\circ} 2$

Cantidad de intervenciones hechas por cada tema $y$ momentos en que se hicieron

\begin{tabular}{|c|c|c|c|c|c|}
\hline Tópicos & $\begin{array}{c}\text { Ocasiones } \\
\text { en las que se } \\
\text { intervinieron }\end{array}$ & $\begin{array}{c}\text { Cantidad de } \\
\text { intervenciones }\end{array}$ & $\begin{array}{c}\text { \% que } \\
\text { representa la } \\
\text { muestra del total }\end{array}$ & $\begin{array}{c}\text { Línea } \\
\text { Tiempo } \\
\text { (min) }\end{array}$ & $\begin{array}{c}\text { Tiempo } \\
\text { usado } \\
\text { (min) }\end{array}$ \\
\hline 1 & Primera & 4 & 4.49 & 0 & 1 \\
\hline 2 & Única & 14 & 15.73 & 1 & 9 \\
\hline 3 & Primera & 9 & 10.11 & 10 & 4 \\
\hline 1 & Segunda & 5 & 5.61 & 14 & 2 \\
\hline 4 & Primera & 8 & 8.98 & 16 & 6 \\
\hline 4 & Segunda & 11 & 12.35 & 22 & 12 \\
\hline 3 & Segunda & 3 & 3.37 & 34 & 2 \\
\hline 4 & Tercera & 7 & 7.86 & 36 & 2 \\
\hline 5 & Primera & 11 & 12.35 & 38 & 9 \\
\hline 4 & Cuarta & 5 & 5.61 & 47 & 2 \\
\hline 5 & Segunda & 5 & 5.61 & 49 & 5 \\
\hline 6 & Única & 7 & 7.86 & 54 & 7 \\
\hline
\end{tabular}
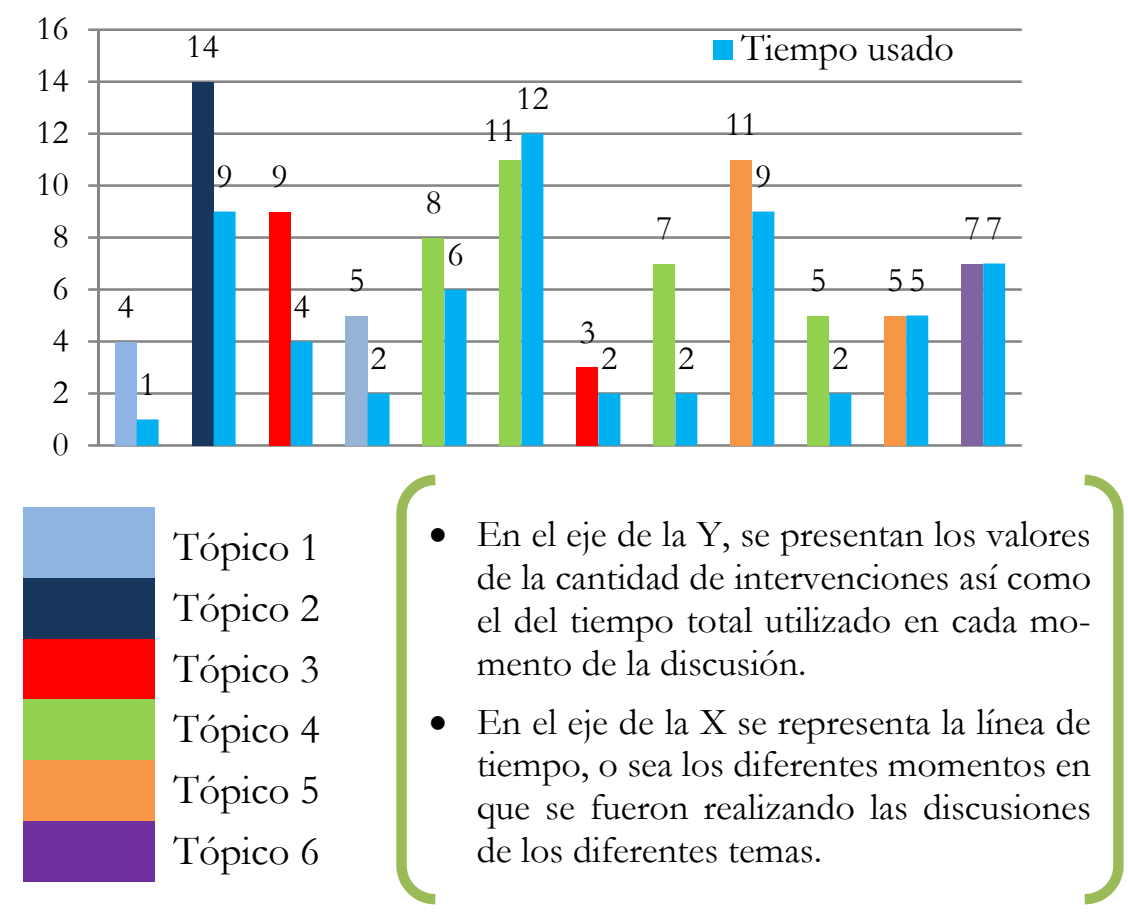
José Antonio Aceituno, Marcos Marmolejos, Evangelista Marte, Pablo Bolivar, Alejandro Aceituno

\section{Etapa de diagnóstico}

Se propone utilizar la técnica conocida como "Sondeo de Problemas", para desarrollar la siguiente fase del trabajo.

\subsection{SONDEO DE PROBLEMAS}

En esta etapa de la investigación y con la información recopilada hasta el momento, se impone que se utilice una técnica que permita acercarnos a la determinación de las causas que han originado las dificultades que impiden que el aprendizaje de la Física se desarrolle adecuadamente, en el nivel de enseñanza que estamos evaluando.

Por tal motivo, seleccionamos al "Sondeo de Problemas", que es una técnica que nos lleva con precisión a aislar las causas de un problema, incluye un análisis del estado actual, comparándolo con lo que debía estar ocurriendo y es el grupo el que redefine el problema y deduce sus causas.

Para este fin, se coordinó una reunión con los mismos integrantes del grupo que se había conformado días antes para aplicar la técnica de "Grupo Central".

Como en la técnica anterior, el moderador fue el profesor que a su vez es el investigador que realiza este trabajo, y los registros fueron tomados en la pizarra y en vídeo.

La distribución de los sujetos dentro del local se representa en el esquema siguiente: (vista superior). 


\section{Esquema N. ${ }^{\circ} 2$}

Ubicación en el aula de los participantes en el Sondeo de Problemas

La "P" representa al profesor y los números a cada uno de los sujetos que participan en la reunión, tal y como se les asignó en la reunión del "Grupo Central" y consta en las páginas anteriores en este propio artículo.

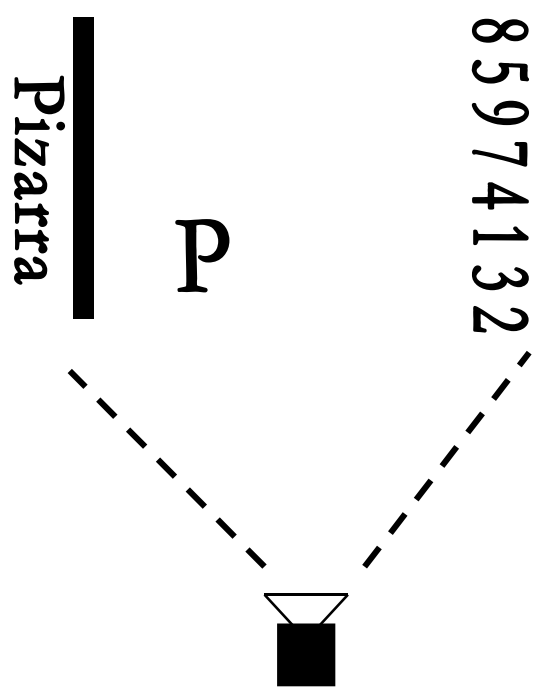

El horario escogido fue negociado con anterioridad, teniendo en cuenta el tiempo disponible de todos los integrantes. Se acordó que fuera después de terminadas las clases de la sesión de la mañana, y como local fue escogido, el Salón de Reuniones del Área de Ciencias Básicas y Ambientales del INTEC, que cuenta con muebles cómodos, buena iluminación y está climatizada.

La reunión puede considerarse dividida en tres etapas. En la primera el profesor (moderador) presenta el estado inicial de la situación, para esto se auxilió de las reflexiones a modo de síntesis que se escribieron al final del informe de lo recogido en la técnica de "Grupo Central", además del gráfico presentado anteriormente, poniendo a disposición de todos la apreciación que tuvo el investigador acerca de la frecuencia con que fueron debatidos los diferentes temas y la intensidad con que se hizo en cada caso. 
José Antonio Aceituno, Marcos Marmolejos, Evangelista Marte, Pablo Bolivar, Alejandro Aceituno

Debe comentarse que la totalidad de los miembros del grupo estuvieron de acuerdo con estas apreciaciones, y se manifestaron a favor de que como se les había presentado recogían el sentimiento que quisieron expresar, y que organizadas así les propicia una mejor comprensión de la situación.

En la segunda etapa, se inicia la sesión de trabajo del grupo para llegar al análisis de las causas. Aquí el moderador utilizó una pizarra para registrar y organizar la información. La dividió al medio con una línea vertical de extremo a extremo, con el objetivo de usar una parte para analizar las acciones del profesor y la otra para analizar las acciones del alumno que se llevan a cabo mientras se desarrollan el proceso de Enseñanza-Aprendizaje de la Física. Después de negociar con los integrantes del grupo, por sobre cuál de los dos comenzar a emitir criterios (por el profesor o por el alumno), decidieron comenzar por el profesor, y entonces el moderador le propone que los criterios se emitan considerando dos interrogantes: ¿Qué hace? y ¿qué debia hacer?, con lo que estuvieron totalmente de acuerdo.

Para facilitar los registros, se subdividió la pizarra en varias partes más, como se muestra en el siguiente esquema:

\begin{tabular}{|c|c|c|c|}
\hline \multicolumn{2}{|c|}{ Profesor } & \multicolumn{2}{|c|}{ Alumno } \\
\hline ¿Qué hace? & ¿Qué debía hacer? & ¿Qué hace? & ¿Qué debía hacer? \\
\hline & & & \\
& & & \\
& & & \\
\hline
\end{tabular}


Veamos ahora cuáles fueron los planteamientos textuales que surgen después de haber hecho la introducción del tema (primera etapa). Aparecerán reflejados, tal y como se recogieron en la pizarra, organizados en las columnas que se construyeron a raíz de la propuesta del investigador de reflexionar acerca de las acciones del profesor y del alumno en diferentes momentos de la clase de Física.

Para una mayor comodidad y legibilidad plasmaremos en tablas separadas lo concerniente al profesor y lo concerniente al alumno.

\begin{tabular}{|c|c|}
\hline \multicolumn{2}{|c|}{ Profesor } \\
\hline ¿Qué hace? & ¿Qué debe hacer? \\
\hline $\begin{array}{l}\text { - Imparte la teoría, menciona las } \\
\text { Leyes y pone las formulas corres- } \\
\text { pondientes a cada ley. } \\
\text { - Resuelve problemas del libro a } \\
\text { partir de las fórmulas que pre- } \\
\text { sentó en la teoría. } \\
\text { - Deja muchos problemas para } \\
\text { resolver como tarea. } \\
\text { - En la próxima clase, revisa la } \\
\text { tarea, pero no toda porque no da } \\
\text { tiempo y hay que continuar con el } \\
\text { programa. }\end{array}$ & $\begin{array}{l}\text { - Impartir la teoría a partir de los } \\
\text { experimentos que hicieron los cien- } \\
\text { tíficos en sus épocas, y que los lleva- } \\
\text { ron a descubrir las leyes de la Física. } \\
\text { Usar experimentos demostrativos. } \\
\text { - Demostrar de donde salen las } \\
\text { fórmulas } \\
\text { - Resolver los problemas haciendo } \\
\text { un análisis físico primero y después } \\
\text { deducir que fórmula o fórmulas usar } \\
\text { en cada situación problémica. } \\
\text { - Desarrollar más cantidad de } \\
\text { problemas en el aula en elaboración } \\
\text { conjunta con los alumnos, y tratar } \\
\text { que sean relacionados con la vida } \\
\text { cotidiana. } \\
\text { - Desarrollar más cantidad de Prác- } \\
\text { ticas de Laboratorio por cada uno } \\
\text { de los temas impartidos, para lograr } \\
\text { una mejor vinculación de la teoría } \\
\text { con la práctica. }\end{array}$ \\
\hline
\end{tabular}


José Antonio Aceituno, Marcos Marmolejos, Evangelista Marte, Pablo Bolivar, Alejandro Aceituno

\begin{tabular}{|c|c|}
\hline \multicolumn{2}{|c|}{ Alumno } \\
\hline ¿Qué hace? & ¿Qué debe hacer? \\
\hline $\begin{array}{l}\text { - Estudiar por las notas de clase. } \\
\text { - Memorizar fórmulas para re- } \\
\text { solver los problemas. } \\
\text { - Limitarse a lo que da el profesor. } \\
\text { - Realizar escasas Prácticas de } \\
\text { Laboratorio. }\end{array}$ & $\begin{array}{l}\text { - Estudiar más por el libro de texto. } \\
\text { - Tratar de no memorizar las fór- } \\
\text { mulas, obtenerlas a partir de razo- } \\
\text { namientos físicos y con ayuda del } \\
\text { álgebra. } \\
\text { - Investigar la vinculación que tienen } \\
\text { los contenidos impartidos con la } \\
\text { vida cotidiana. } \\
\text { - Realizar proyectos de investigación } \\
\text { para vincular la teoría con la práctica. }\end{array}$ \\
\hline
\end{tabular}

A partir del análisis de los resultados anteriores, nos dimos a la tarea de comenzar a diseñar y construir las primeras habitaciones de lo que será La mansión de la física, un video juego en tres dimensiones que se propone como una alternativa para dar solución a las carencias explicitadas a través de las necesidades educativas que emergen de la aplicación de las técnicas precedentes.

El primer tema seleccionado fue el de la Cinemática de la Traslación, tal y como aparece en el curriculum, tanto de la enseñanza media como de la enseñanza a nivel superior.

En la primera habitación se trabajará con el movimiento unidireccional, específicamente el movimiento rectilíneo uniformemente acelerado en la dirección horizontal.

En la segunda habitación, se trabajará con el movimiento rectilíneo uniformemente acelerado en la dirección vertical, donde la aceleración que actúa es la de la gravedad. 
Ya en la tercera y cuarta habitaciones, se trabajará el movimiento bidimensional, que tiene como principal protagonista el movimiento de proyectiles, combinado con el unidimensional, haciendo énfasis en la simultaneidad de los movimientos.

En una quinta habitación, empezaremos a trabajar con la Dinámica, o sea la parte de la Física Mecánica que estudia la interacción de los cuerpos a través de los diferentes tipos de fuerzas.

Estos temas fueron seleccionados a partir del análisis del programa de la asignatura Física I, donde forman parte de los contenidos iniciales con los cuales el alumno debe ir desarrollando determinadas competencias que le permita ir comprendiendo las leyes fundamentales de la Física Mecánica y capacitándose para comprender y transformar el universo que le rodea a partir de las propias leyes y conceptos de esta disciplina

Cada uno de los temas se irán presentando con una vinculación muy estrecha con la vida cotidiana, y las soluciones a los problemas presentados no serán evidentes, sino que el alumno jugador deberá obtener las expresiones de trabajo y calcular los parámetros que necesita para resolver las situaciones. Se deja un margen muy pequeño a las respuestas aleatorias, o al ensayo error.

El jugador contara con una ayuda, que le permitirá encontrar los fundamentos teóricos que necesita para deducir o recordar las ecuaciones que utilizara en la búsqueda de las soluciones. En ningún caso esta ayuda dará soluciones directas, es más bien como el profesor orientador que lo guía y le da herramientas para que trabaje.

Queda incluida la posibilidad de que la ayuda lleve al jugador a investigar o buscar información, sobre la situación física que los ocupa, en libros de texto, internet, etc. 
José Antonio Aceituno, Marcos Marmolejos, Evangelista Marte, Pablo Bolivar, Alejandro Aceituno

\section{Conclusiones}

A partir del análisis de los resultados que tenemos después de aplicar las diferentes técnicas, podemos concluir que las principales carencias de los estudiantes que ingresan al INTEC y que reciben Física en sus carreras apuntan de manera general a la insuficiente discusión teórica sobre los fenómenos físicos y sus leyes, a esto se suma la poca o ninguna utilización de experimentos demostrativos en el aula, a la constante solución de problemas a partir de la utilización de fórmulas predeterminadas para calcular cada variable específica, además de que no se relacionan suficientemente los contenidos que se imparten con los fenómenos de la vida cotidiana así como al poco o ningún desarrollo de Prácticas de Laboratorio. Sumándole a todo esto el hecho de que son muy pocas las horas en los programas académicos del bachillerato que se le dedica a la impartición de la física.

\section{Recomendaciones}

Deben validarse la utilización de La mansión de la física, como recurso didáctico que se enfoca en tratar de dar solución a las carencias detectadas en los alumnos. Esta validación debe estar enfocada en la propia estructura del video juego, así como en las posibles maneras de utilizarlo con los estudiantes, que pudiera comenzarse por los siguientes aspectos:

- Indagar la conveniencia de que los alumnos utilicen este recurso antes, durante o después de haber recibido los contenidos en clase. ¿En qué momento el alumno puede jugar?, ¿en qué momento es más efectivo?

- En el caso de ser utilizado en clases, ¿en qué tipo de actividad docente podría utilizarse el juego?, ¿qué estructura debe tener?

- ¿Podría diseñarse el juego de forma tal que pueda usarse como evaluación de los aprendizajes? 
Algunas carencias detectadas en los estudiantes que ingresan a INTEC y reciben Física General en sus carreras

\section{Referencias bibliográficas}

Adams, W. K. (2010). Student Engagement and Learning with PhET Interactive Simulations, Multimedia in Physics Teaching and Learning Proceedings. Il Nuovo Cimento C, 3, 21-32. Recuperado http://en.sif.it/journals/ncc/econtents/ 2010/033/03/article/25 Doi: 10.1393/ncc/i2010-10623-0

Adams, W. K.; Reid, S.; LeMaster, R.; McKagan, S. B.; Perkins, K. K.; Dubson, M. \& Wieman, C. E. (2008). A Study of Educational Simulations Part II-Interface Design. Journal of Interactive Learning Research, 19(4), 551-577.

Adams, W. K.; Reid, S.; LeMaster, R.; McKagan, S. B.; Perkins, K. K.; Dubson, M. and Wieman, C. E. A Study of Educational Simulations Part I - Engagement and Learning. (2008). Journal of Interactive Learning Research, 19(3), 397-419.

Adams, W. K.; Paulson, A. \& Wieman, C. E. What Levels of Guidance Promote Engaged Exploration with Interactive Simulations? (2009). [Video en línea]. Recuperado de https://phet.colorado.edu/es/research

Arias, M.; Romero, V. \& Pérez, V. (2011). Informe física general I. Santo Domingo: Instituto Tecnológico de Santo Domingo.

Attali, J.; Abreu, R.; Álvarez, R. R.; Arnould, C.; Asilis, C.; Corripio, J. L.; Lemoine M.; Owens, J.; Pezzini, M.; Prats, E. J.; Stubbe, M. \& Whitman, I. (2010). Informe de la Comisión Internacional para el Desarrollo Estratégico de la República Dominicana. Santo Domingo: Ministerio de Economía Planificación y Desarrollo.

Beichner, R. J. (1994). Testing student interpretation of kinematics graph. American Association of Physics Teachers, 62(8), 751-753. 
José Antonio Aceituno, Marcos Marmolejos, Evangelista Marte, Pablo Bolivar, Alejandro Aceituno

Christian, W.; Esquembre, F.; Barbato, L. (2011). Spore Award: Open Source Physics. Recuperado de http://www.compadre. org/portal/

Clements, P. \& Northrop, L. (2002). Software Product Lines: Practices and Patterns. Massachusetts: Addison-Wesley.

Esquembre, F.; Martin, E.; Christian, W.; Belloni, M. (2004). Enseñanza de la física con material interactivo. Madrid: Pearson Education.

Filmer, D.; Hasan, A. \& Pritchett, L. (2006). A Millennium Learning Goal: Measuring Real Progress in Education-Working [Paper 97]. Recuperado de http://www.cgdev.org/files/9815_file_ WP97.pdf

Hausmann, R.; Hidalgo, C. A.; Jiménez, J. A.; Lawrence, R.; LevyYeyati, E.; Pritchett, L.; Sabel, C. \& Schydlowsky, D. (2012). Construyendo un mejor futuro para la República Dominicana: Herramientas para el desarrollo. [Working Papers 38]. Massachusetts: Center for the International Development at Harvard University.

Hestenes, D.; Wells, M. \& Swackhamer, G. (1992). Force concept inventory. The Physics Teacher, 30, 141-158. Recuperado de http:/ / ptc.weizmann.ac.il/7A9C98E5-6499-4C6F-AD0D4353A7A92A1C/FinalDownload/DownloadId-B2997929 F41F6FEAF78C9C338638632B/7A9C98E5-6499-4C6FAD0D-4353A7A92A1C/_Uploads/dbsAttachedFiles/ 1852FCI.pdf

Hsu, L. \& Heller, K. (2004). AIP Conference Proceedings. Recuperado de http://groups.physics.umn.edu/physed/Talks/ PERC\%202013_final.pdf

Podolefsky, N. S.; Perkins, K. K. \& Adams, W. K. (2010). Factors promoting engaged exploration with computer simulations. Recuperado de http://journals.aps.org/prstper/abstract/10.1103/Phys RevSTPER.6.020117 


\section{José Antonio Aceituno}

Investigador principal de este artículo. Licenciado en Física; Master en Ciencias de la Educación Superior. Egresado de la Universidad Central de Las Villas, Cuba; profesor del Área de ciencias Básicas y Ambientales del Instituto Tecnológico de Santo Domingo (INTEC) desde el 2006.

Email: jose.aceituno@intec.edu.do

\section{Marcos Marmolejos}

Asesor. Es egresado de INTEC. Ingeniería Electrónica y Telecomunicaciones del Instituto Tecnológico de Santo Domingo (INTEC).

Email: marco.marmolejos@gmail.com

\section{Evangelista Marte}

Desarrollador. Es estudiante de término de la carrera de Ingeniería Civil del Instituto Tecnológico de Santo Domingo (INTEC).

Email: evangelista.399@gmal.com

\section{Pablo Bolívar}

Desarrollador. Estudiante de término de la carrera de Ingeniería en Sistemas del Instituto Tecnológico de Santo Domingo (INTEC).

Email: pablobolivar joa@gmail.com 
José Antonio Aceituno, Marcos Marmolejos, Evangelista Marte, Pablo Bolivar, Alejandro Aceituno

\section{Alejandro Aceituno}

Especialista en Estadística. Egresado de INTEC.

Licenciado en Matemáticas con concentración en

Estadísticas y Ciencias Actuariales.

Email: aceitunoalvarez@hotmail.com

Recibido: 05/02/2014 Aprobado: 23/10/2014

758 Ciencia y Sociedad 2014; 39(4): 731-758 